Aus diesem Vergleich ergibt sich einerseits der große Unterschied zwischen der Zusammensetzung von Boletus Bellini und Boletus edulis (Steinpilz) hinsichtlich des Gehaltes an Stickstoff-Substanz, andererseits aber die Übereinstimmung aller Zahlen für die Bestandteile bei den verschiedenen verwandten Boletus-Arten, die eine einzige Gruppe bilden, wie z. B. Boletus granulatus, bovinus, elegans, luteus und Boletus Bellini, eine Gruppe, deren besonderes charakteristisches Merkmal der verhältnismäßig niedrige Gehalt an Stickstoff-Substanz im Vergleich zum Boletus edulis ist.

Zum Schluß will ich noch anführen, daß Boletus Bellini ebenso wie die anderen oben erwähnten Arten, obwohl er dem Boletus edulis in bezug auf Aroma weit nachsteht, es verdient, bekannt zu werden, und sich namentlich zum Genuß in trockenem Zustand, als Gewürz, eignet. Boletus Bellini verdiènt daher in die Reihe der eBbaren Pilze eingestellt zu werden und zwar um so mehr, als er gerade in einer Jahreszeit vorkommt, in der die anderen, mehr geschätzten Pilze schon lange nicht mehr vorhanden sind.

Was insbesondere die trockenen Pilze betrifft, so sind die meisten unserer lokalen hygienischen Verordnungen vielleicht etwas zu streng, da sie im allgemeinen nur den Boletus edulis zulassen. Berechtigt ist dagegen die Vorschrift, welche den Verkauf von Mischungen von trockenen Pilzen verschiedener Arten verbietet, da eine Duldung in dieser Hinsicht zu schweren Mißständen Veranlassung geben könnte, indem sich nämlich mancher Händler veranlaßt sehen könnte, alle möglichen Arten, auch von scbädlichen getrockneten Pilzen, zu verkaufen, wie uns dies die Erfahrung lehrt. Dagegen erscheint mir der vollständige Ausschluß des Verkaufes der oben erwähnten Pilzarten nicht gerechtfertigt, da diese in jeder Hinsicht verdienen, zum Verbrauch zugelassen $\mathrm{zu}$ werden, und ebenso leicht auch im trockenen Zustand identifiziert werden können wie Boletus edulis.

Januar 1907.

\title{
Beiträge zur Kenntnis des Aschengehaltes des Paprika.
}

\section{Von}

\section{Dr. Richard Windisch.}

Mitteilung aus der Agrikulturchemischen Versuchsstation der landwirtschaftlichen Akademie in Keszthely (Ungarn).

Den Fachgenossen, welche sich eingehender mit der Untersuchung der im Handel befindlichen Paprikasorten beschäftigt haben, ist es gewiß nur zu gut bekannt, wie außerordentlich schwankend die Literaturangaben über den Aschengehalt des Paprika sind.

Ball $6^{1}$ ), sowie Bujard und Baier ${ }^{2}$ ) geben als höchsten Aschengehalt von reinem Paprika $5 \%$, Bittó ${ }^{3}$ ) gibt 5 bis $6,50 \%$, Vog $1^{4}$ ) und H. Röttger ${ }^{5}$ ) $6,50 \%$,

1) Mátyás Balló, Budapest székes fơváros vegyészeti és tápszervizsgáló intézetének evkönyve 1895 , S. 87. 1894 , S. 81 .

2) A. Bujard und E. Baier, Hilfsbuch für Nahrungsmittelchemiker. Berlin, J. Springer,

3) Landwirtschaftliche Versuchsstationen 1896, 46, 309.

4) A. E. Vogl, Die wichtigsten vegetabilischen Nahrungs- und Genufmittel, Wien 1899,

5) H. R öttger, Lehrbuch dex Nahrungsmittelchemie. Leipzig, J. A. Barth, 3. Aufl., 1907, S. 486. - Die erste Auflage dieses Buches (1894, S. 259) bezeichnete als höchst zulässigen A s chengehalt $5 \%$. 
V. Vedrödi $\mathrm{i}^{1}$ ) bis $6,50 \%$ und Strohmer $\left.{ }^{2}\right) 5$ bis $6 \%$ an. In den deutschen „Vereinbarungen"3 3 ist als höchste Grenzzahl für lufttrockenen Paprika 6,50\% Asche festgesetzt. Die schweizerischen Chemiker schlagen nur 5\% Asche vor. Im Entwurfe von A. Vogl und T. F. Han a usek für den Codex alimentarius Austriacus (Kapitel "Gewürze") wird ein Aschengehalt von 5 bis $6 \%$ gefordert. Beythien ${ }^{4}$ ) fand in 32 Proben Paprika des Dresdener Handels im Mittel 6,34\% Asche; der höchste Gehalt war $7,76 \%$, der niedrigste $5,35 \%$.

Allen diesen Literaturangaben stebt diejenige von Georg Gregor ${ }^{5}$ ) gegenüber, welcher auf Grund seiner diesbezüglichen Untersuchungen den höchst zulässigen Aschengehalt des Paprikas auf 10\% erhöht wissen will. Gregor faßt das Ergebnis seiner Untersuchungen dahin zusammen, daf aus einem erhöhten Aschengehalt allein noch nicht auf eine Verfälschung der Paprikapulver geschlossen werden darf.

A. G. Stillwe $11^{6}$ ), welcher in neuester Zeit Paprikapulver-Untersuchungen in New-York ausführte, wobei er sich eines Materiales von authentischer Reinheit bediente, teilt die Paprikasorten in drei Gruppen ein:

I. Hochwertige (milde) nur aus der Fruchtschale bestehende Paprikasorten. Gesamtasche $7-8 \%$.

II. Mittlere etwas Samen enthaltende Qualitäten. Gesam tasche 6-7\%.

III. Minderwertige Sorten, die praktisch nur aus Samen und Stengeln bestehen. Gesamtasche $9-13 \%$.

Die Paprikafrüchte bestehen aus verschiedenen Teilen, welche sich ziemlich leicht voneinander trennen lassen und einen sehr verschiedenen Aschengehalt besitzen. Die Menge dieser verschiedenen Teile der Paprikafrüchte schwankt auch innerhalb ziemlich weiter Grenzen. Behufs Herstellung von gemahlenem Paprika werden die Früchte sehr verschiedenen Behandlungen unterworfen; gewisse Teile derselben werden entfernt, die verschiedenen Bestandteile in sehr verschiedenen Verhältnissen gemischt und miteinander vermahlen, wodurch sich auch der schwankende Aschengehalt der im Handel befindlichen Paprikasorten erklärt.

Im vergangenen Jahre habe ich Gelegenheit gehabt, den Aschengehalt von 20 unzweifelhaft reinen Paprikaproben zu bestimmen. Die untersuchten Proben wurden von mir selbst eigenhändig aus den Früchten dargestellt.

In erster Linie erhielt ich von Professor Kerpely an der landwirtschaftlichen Akademie in Debrecen 18 verschiedene Sorten von Paprikafrüchten, welche aus der Ernte des Jahres 1904 stammten und ron dem guten Sandboden des Versuchsfeldes, das in guter Kraft war, geerntet wurden. Im Herbste des Jahres 1904 traten in Debrecen Frühfröste ein, wodurch ein Teil der Früchte kurz vor der Ernte teilweise erfror, infolgedessen die Samen ibre Keimkraft verloren. Die Früchte waren trotz des Gefrierens, nachdem sie nach der Ernte langsam aufgetaut und dann in der heizbaren

1) Zeitschrift für Nahrungsmitteluntersuchung, Hygiene und Warenkunde 1893, 7, 385.

2) Vergl. Béla v. Bittó, Landwirtschaftliche Versuchsstationen 1893, 42, 309.

3) Vereinbarungen zur einheitlichen Untersuchung und Beurteilung von Nahrungs- und Genußmitteln sowie Gebrauchsgegenständen für das Deutsehe Reich II, S. 55.

4) Diese Zeitschrift 1902, 5, 858 .

5) Diese Zeitschrift 1900, 3, 460.

6) Journ. Amer. Chem. Soc. 1906, 28, 1608; Chemisches Zentralblatt 1907, I, 130. 
amerikanischen Tabaktrockenstube ausgetrocknet worden waren, in Habitus, Aussehen und Farbe vollkommen normal.

Das Jahr 1904, schreibt Prof. Kerpely, war dem Anbau der Paprikapflanze nicht günstig. Der Sommer war äußerst trocken und heiß, wodurch die Entwickelung derselben gehemmt wurde; dadurch fiel die Reifezeit in den Herbst und die Septemberfröste richteten einen Schaden an. Nachdem der Frost die Früchte knapp vor der Ernte befiel, konnten dieselben als normal entwickelt bezeichnet werden. Ich erhielt die Früchte Mitte Oktober 1905 und konnte an ihnen weder in der Farbe noch in der Entwickelung eine Abnormität bemerken. Daß die Samen ihre Keimkraft verloren hatten, war ja ohnedies kein Hindernis für die Aschenbestimmungen.

Die einzelnen Früchte wurden nun mittels Gänsefedern gut abgebürstet, der Kelch und der Stengel sorgfältig von dem Fruchtfleisch, den Samenträgern und Samen getrennt. Die Fruchtwand wurde mittels einer Schere zerschnitten und samt den Samen und Samenträgern 24. Stunden lang bei einer Temperatur von beiläufig $60-70^{\circ} \mathrm{C}$ getrocknet. Die so vorbereiteten einzelnen Sorten wurden dann auf einer Excelsior-Mühle fein gemahlen und diese Proben dienten darauf zur Aschenbestimmung.

Zum Zwecke der Aschenbestimmung wurde die eine genat abgewogene Menge Paprika (etwa 4,5 g Trockensubstanz) enthaltende Platinschale auf einem mit Asbest überzogenen Drahtnetze so lange erhitzt, bis der größte Teil der Substanz verkohlt war und sich keine zum Husten reizenden Dämpfe mehr entwickelten. Der verkohlte Rückstand wurde dann über freier Flamme vollkommen verascht und während dieser Zeit öfters mit einem dicken Platindrahte umgerührt. Bei den meisten Proben genügte dieses Verfahren. In Ausnahmefällen wurde der abgekühlte verkohlte Rückstand mit destilliertem Wasser angefeuchtet, auf dem Wasserbade eingetrocknet und schlieblich gänzlich verascht.

Die Tabelle auf S. 392 und 393 enthält die bei den 18 von Professor Kerpely übersandten Paprikaproben erhaltenen Untersuchungsergebnisse, sowie ferner Angaben über die Form der Früchte, ihre mittlere Länge und Breite in Millimetern, und die Anzahl der von einer Sorte im ganzen eingesandten Früchte. Die Länge und Breite wurde an allen eingesandten Früchten gemessen, die Angaben, welche in der entsprechenden Spalte der Tabelle sich befinden, bilden das Mittel der einzelnen Messungen. In jeder Probe wurde der Aschengehalt in zwei getrennten Partien bestimmt. Der Aschengehalt der vor dem Vermahlen entfernten Kelche und Stengel wurde ebenfalls bestimmt. Die Aschenbestimmung des Paprikapulvers geschah in der Trockensubstanz, die der vereinigten Kelche und Stengel in der lufttrockenen Substanz.

Wie aus der Tabelle ersichtlich ist, betrug der niedrigste Aschengehalt in der Trockensubstanz des Paprika 6,53\%, der höchste 9,51\%; das Mittel aus sämtlichen Bestimmungen berechnet sich zu $8,36 \%$. Da sich jedoch die Literaturangaben betreffs des Aschengehaltes des Paprikapulvers höchstwahrscheinlich nicht auf Trockensubstanz beziehen, wurde der Aschengehalt auch auf lufttrockene Substanz berechnet. Bei dieser Umrechnung wurde der Wassergehalt des Paprikas auf Grund der Untersuchungen von Bitto und Beythien ${ }^{1}$ ) zu 10\% angenommen. Bei diesem Wassergehalte würde sich für den Aschengehalt der lufttrockenen Paprikamuster als niedrigster 


\begin{tabular}{|c|c|c|c|c|c|}
\hline \multirow{2}{*}{ No. } & \multirow{2}{*}{ Name des Paprikas } & \multirow{2}{*}{ Beschreibung der Fruchtîorm } & \multicolumn{2}{|c|}{$\begin{array}{c}\text { Mittlere Länge } \\
\text { und Breite } \\
\text { der Früehte }\end{array}$} & \multirow{2}{*}{$\begin{array}{l}\text { Anzahl } \\
\text { der } \\
\text { unter- } \\
\text { suehten } \\
\text { Früthte }\end{array}$} \\
\hline & & & $\begin{array}{c}\text { Länge } \\
\mathrm{mm}\end{array}$ & $\begin{array}{c}\text { Breite } \\
\mathrm{mm}\end{array}$ & \\
\hline 1 & Cardinal-Paprika & $\begin{array}{l}\text { Schmale, längliche, an der Spitze } \\
\text { etwas eingebogene Früchte }\end{array}$ & 181,0 & 27,1 & 10 \\
\hline 2 & Cardinal-Paprika & Wie bei No. 1 & 206,8 & 28,1 & 11 \\
\hline 3 & $\begin{array}{l}\text { Roter sehr dünner } \\
\text { Cayenne-Paprika }\end{array}$ & $\begin{array}{c}\text { Längliche spitze Früchte, von der } \\
\text { Dicke eines Fingers }\end{array}$ & 128,3 & 16,0 & 21 \\
\hline 4 & Celestial & $\begin{array}{l}\text { Beiläufig nufgroße Früchte mit teil- } \\
\text { weise spitzen und stumpfen Enden }\end{array}$ & 50,0 & 30,0 & 15 \\
\hline 5 & $\begin{array}{l}\text { Kleiner schmaler roter } \\
\text { chilenischer Paprika }\end{array}$ & Schmale kurze spitze Früchte & 79,3 & 12,6 & 22 \\
\hline 6 & $\begin{array}{l}\text { Kirschenförmiger roter } \\
\text { peruaner Paprika }\end{array}$ & $\begin{array}{l}\text { Früchte von der Form und Größse } \\
\text { einer großen Kirsche }\end{array}$ & 35,0 & 20,0 & 26 \\
\hline 7 & Elefantenrüssel-Paprika & $\begin{array}{l}\text { Lange, dünne, an der Spitze etwas } \\
\text { eingebogene Früchte }\end{array}$ & 195,0 & 26,0 & 11 \\
\hline 8 & Madelun-Paprika & Kurze dicke Früchte & 76,0 & 39,0 & 12 \\
\hline 9 & $\begin{array}{l}\text { Mauthner's gelber } \\
\text { Paprika }\end{array}$ & $\begin{array}{c}\text { Kegelförmige Früchte von der Größe } \\
\text { einer Kindsfaust }\end{array}$ & 77,0 & 51,0 & 11 \\
\hline 10 & Süßer Riesen-Paprika & Längliche spitze Früchte & 138,0 & - & 11 \\
\hline 11 & $\begin{array}{l}\text { Roter tomatenförmiger } \\
\text { Paprika }\end{array}$ & $\begin{array}{l}\text { Von der Form und Gröfe einer } \\
\text { kleinen Tomatenfrucht }\end{array}$ & - & - & 12 \\
\hline 12 & $\begin{array}{c}\text { Grofer langer spanischer } \\
\text { Paprika }\end{array}$ & $\begin{array}{c}\text { Spitze aber nicht übermäßig lange } \\
\text { Früchte }\end{array}$ & 110,0 & 31,0 & 13 \\
\hline 13 & $\begin{array}{l}\text { Gelber tomatenförmiger } \\
\text { Paprika }\end{array}$ & $\begin{array}{l}\text { Form der Frucht wie bei No. 11, } \\
\text { Größe beiläufig wie eine Kindsfaust }\end{array}$ & - & - & 12 \\
\hline 14 & Dicker eckiger Paprika & $\begin{array}{l}\text { Frucht gerunzelt und tief gefurcht, } \\
\text { von der Gröfe einer Kindsfaust }\end{array}$ & 68,8 & 52,3 & 10 \\
\hline 15 & $\begin{array}{l}\text { Dicker eckiger roter } \\
\text { Paprika }\end{array}$ & $\begin{array}{l}\text { Früchte kindsfaustgroß, } \\
\text { beinahe gleichmäßig lang und breit }\end{array}$ & 82,3 & 54,1 & 11 \\
\hline 16 & $\begin{array}{l}\text { Grofer eckiger süßer } \\
\text { roter Paprika }\end{array}$ & $\begin{array}{l}\text { Früchte kindsfaustgrof mit gefurchter } \\
\text { Oberfläche, mit spitzen a. stumpfen Enden }\end{array}$ & 70,0 & 55,9 & 9 \\
\hline 17 & $\begin{array}{l}\text { Großer eckiger süßer } \\
\text { roter Paprika }\end{array}$ & Wie No. 16, jedoch größere Früchte & 79,5 & 60,0 & 11 \\
\hline 18 & Szegediner Rosen-Paprika & Schmale längliche Früchte & 99,6 & 24,7 & 13 \\
\hline
\end{tabular}




\begin{tabular}{|c|c|c|c|c|c|c|c|}
\hline \multicolumn{4}{|c|}{ Gemablener Paprika } & \multicolumn{4}{|c|}{ Stengel and Kelehe } \\
\hline \multirow{2}{*}{$\begin{array}{l}\text { Zur Aschen- } \\
\text { bestimmung } \\
\text { abgewogene } \\
\text { Troeken- } \\
\text { substanz }\end{array}$} & \multirow{2}{*}{$\begin{array}{l}\text { Darin } \\
\text { gefundene } \\
\text { Asthen- } \\
\text { menge } \\
\mathrm{g}\end{array}$} & \multicolumn{2}{|c|}{ Asehengehalt } & \multirow{2}{*}{$\begin{array}{c}\text { Zur Aschen- } \\
\text { bestimmung } \\
\text { abgewogene } \\
\text { lufturoekene } \\
\text { Substanz } \\
\mathrm{g} \\
\end{array}$} & \multirow{2}{*}{$\begin{array}{l}\text { Darin } \\
\text { gefundene } \\
\text { Aschen- } \\
\text { menge } \\
\text { g }\end{array}$} & \multicolumn{2}{|c|}{ Asthengehalt } \\
\hline & & $\begin{array}{c}\begin{array}{c}\text { Einzelbe- } \\
\text { stimmungen } \\
0 \%\end{array} \\
\end{array}$ & $\begin{array}{c}\text { Mittel } \\
0_{0} \\
\end{array}$ & & & \begin{tabular}{|c|}
$\begin{array}{c}\text { Einzelbe- } \\
\text { stimmungen } \\
\%\end{array}$ \\
\end{tabular} & $\begin{array}{c}\text { Mittel } \\
0_{0} \\
\end{array}$ \\
\hline $\begin{array}{l}4,6678 \\
4,7509 \\
\end{array}$ & $\begin{array}{l}0,2933 \\
0,3223 \\
\end{array}$ & $\begin{array}{l}6,28 \\
6,78 \\
\end{array}$ & 6,53 & $\begin{array}{l}2,7271 \\
2,6328 \\
\end{array}$ & $\begin{array}{l}0,3372 \\
0,2776 \\
\end{array}$ & $\begin{array}{l}12,36 \\
10,54 \\
\end{array}$ & 11,45 \\
\hline $\begin{array}{l}4,4975 \\
4,4787\end{array}$ & $\begin{array}{l}0,3982 \\
0,3907 \\
\end{array}$ & $\begin{array}{l}8,85 \\
8,72 \\
\end{array}$ & 8,78 & $\begin{array}{l}2,6064 \\
1,9454\end{array}$ & $\begin{array}{l}0,2909 \\
0,2437\end{array}$ & $\begin{array}{l}11,16 \\
12,52 \\
\end{array}$ & 11,84 \\
\hline $\begin{array}{l}4,6539 \\
4,6490\end{array}$ & $\begin{array}{l}0,3766 \\
0,3930\end{array}$ & $\begin{array}{l}8,95 \\
8,45\end{array}$ & 8,70 & $\begin{array}{l}1,5424 \\
1,3576\end{array}$ & $\begin{array}{l}0,2027 \\
0,1603\end{array}$ & $\begin{array}{l}13,14 \\
11,80\end{array}$ & 12,47 \\
\hline $\begin{array}{l}4,8858 \\
4,7825\end{array}$ & $\begin{array}{l}0,3903 \\
0,4120\end{array}$ & $\begin{array}{l}7,98 \\
8,61\end{array}$ & 8,29 & $\begin{array}{l}1,5300 \\
0,8822\end{array}$ & $\begin{array}{l}0,1710 \\
0,0905\end{array}$ & $\begin{array}{l}11,17 \\
10,25\end{array}$ & 10,71 \\
\hline $\begin{array}{l}4,7644 \\
4,7868\end{array}$ & $\begin{array}{l}0,3351 \\
0,3325\end{array}$ & $\begin{array}{l}7,03 \\
6,94\end{array}$ & 6,98 & $\begin{array}{l}1,8592 \\
1,2274\end{array}$ & $\begin{array}{l}0,2280 \\
0,1392\end{array}$ & $\begin{array}{l}12,30 \\
11,34\end{array}$ & 11,82 \\
\hline $\begin{array}{l}4,5357 \\
4,5847\end{array}$ & $\begin{array}{l}0,3712 \\
0,3674\end{array}$ & $\begin{array}{l}8,18 \\
8,01 \\
\end{array}$ & 8,09 & $\begin{array}{l}2,2950 \\
2,4489\end{array}$ & $\begin{array}{l}0,8170 \\
0,3537\end{array}$ & $\begin{array}{l}13,81 \\
14,44\end{array}$ & 14,12 \\
\hline $\begin{array}{l}4,5617 \\
4,6095\end{array}$ & $\begin{array}{l}0,4060 \\
0,4070\end{array}$ & $\begin{array}{l}8,90 \\
8,82\end{array}$ & 8,86 & $\begin{array}{l}3,6410 \\
3,2971\end{array}$ & $\begin{array}{l}0,4410 \\
0,3838\end{array}$ & $\begin{array}{l}12,11 \\
11,64\end{array}$ & 11,87 \\
\hline $\begin{array}{l}4,5082 \\
4,4490 \\
\end{array}$ & $\begin{array}{l}0,4147 \\
0,4380\end{array}$ & $\begin{array}{l}9,19 \\
9,84 \\
\end{array}$ & 9,51 & $\begin{array}{l}3,6870 \\
3,8688\end{array}$ & $\begin{array}{l}0,4286 \\
0,4281\end{array}$ & $\begin{array}{l}11,62 \\
12,46\end{array}$ & 12,04 \\
\hline $\begin{array}{l}4,6325 \\
4,5314\end{array}$ & $\begin{array}{l}0,3965 \\
0,3684\end{array}$ & $\begin{array}{l}8,55 \\
8,12\end{array}$ & 8,33 & $\begin{array}{l}2,5572 \\
1,8863\end{array}$ & $\begin{array}{l}0,3415 \\
0,2646\end{array}$ & $\begin{array}{l}13,45 \\
14,02\end{array}$ & 13,73 \\
\hline $\begin{array}{l}4,4687 \\
4,5180\end{array}$ & $\begin{array}{l}0,3852 \\
0,3980\end{array}$ & $\begin{array}{l}8,61 \\
8,80\end{array}$ & 8,70 & $\begin{array}{l}2,7165 \\
3,1025\end{array}$ & $\begin{array}{l}0,3243 \\
0,3540\end{array}$ & $\begin{array}{l}11,86 \\
11,41 \\
\end{array}$ & 11,63 \\
\hline $\begin{array}{l}4,5622 \\
4,6555\end{array}$ & $\begin{array}{l}0,3280 \\
0,3125\end{array}$ & $\begin{array}{l}7,18 \\
6,71\end{array}$ & 6,94 & $\begin{array}{l}2,6513 \\
2,1757\end{array}$ & $\begin{array}{l}0,3358 \\
0,2655\end{array}$ & $\begin{array}{l}12,66 \\
12,20\end{array}$ & 12,43 \\
\hline $\begin{array}{l}4,8562 \\
4,8270\end{array}$ & $\begin{array}{l}0,3952 \\
0.3825\end{array}$ & $\begin{array}{l}8,13 \\
7,92\end{array}$ & 8,02 & $\begin{array}{l}1,9397 \\
2,2005\end{array}$ & $\begin{array}{l}0,2222 \\
0,2395\end{array}$ & $\begin{array}{l}11,45 \\
16,88 \\
\end{array}$ & 11,16 \\
\hline $\begin{array}{l}4,6460 \\
4,6881 \\
\end{array}$ & $\begin{array}{l}0,3795 \\
0,3981 \\
\end{array}$ & $\begin{array}{l}8,16 \\
8,38\end{array}$ & 8.27 & $\begin{array}{l}3,6505 \\
3,3119\end{array}$ & $\begin{array}{l}0,3748 \\
0,3807\end{array}$ & $\begin{array}{l}10,26 \\
11,49\end{array}$ & 10,87 \\
\hline $\begin{array}{l}4,6220 \\
4,5877 \\
\end{array}$ & $\begin{array}{l}0,4048 \\
0,4397 \\
\end{array}$ & $\begin{array}{l}8,75 \\
9,58\end{array}$ & 9,15 & $\begin{array}{l}3,3648 \\
3,8700\end{array}$ & $\begin{array}{l}0,4483 \\
0,5393 \\
\end{array}$ & $\begin{array}{l}13,32 \\
13,77 \\
\end{array}$ & 13,54 \\
\hline $\begin{array}{l}4,6446 \\
4,7528 \\
\end{array}$ & $\begin{array}{l}0,4119 \\
0,4006 \\
\end{array}$ & $\begin{array}{l}8,86 \\
8,42\end{array}$ & 8,64 & $\begin{array}{l}2,4245 \\
8,2185\end{array}$ & $\begin{array}{l}0,3155 \\
0,3857 \\
\end{array}$ & $\begin{array}{l}13,01 \\
11,98 \\
\end{array}$ & 12,44 \\
\hline $\begin{array}{l}4,5134 \\
4,6172\end{array}$ & $\begin{array}{l}0,4170 \\
0,4470 \\
\end{array}$ & $\begin{array}{l}9,23 \\
9,68\end{array}$ & 9,45 & $\begin{array}{l}4,7845 \\
3,5332\end{array}$ & $\begin{array}{l}0,6278 \\
0,4542\end{array}$ & $\begin{array}{l}13,12 \\
12,85\end{array}$ & 12,98 \\
\hline $\begin{array}{l}4,7162 \\
4,7123\end{array}$ & $\begin{array}{l}0,4082 \\
0,3943\end{array}$ & $\begin{array}{l}8,65 \\
8,36\end{array}$ & 8,50 & $\begin{array}{l}2,3552 \\
1,7530\end{array}$ & $\begin{array}{l}0,3049 \\
0,1739\end{array}$ & $\begin{array}{r}12,91 \\
9,88\end{array}$ & 11,39 \\
\hline
\end{tabular}


[Fortsetzung von S. 391.]

Gehalt 5,87\% und als höchster $8,55 \%$ und als Mittel sämtlicher Bestimmungen $7,52 \%$ ergeben.

Außer den oben erwähnten 18 reinen Paprikamustern standen mir auch noch zwei andere Paprikapulver zur Verfügung, welche aus ausgewäbltem Rohmateriale ebenfalls eigenhändig auf folgende Weise dargestellt wurden:

A. Sorgfältig ausgewählte, schön rotfarbige, reife, zerschnittene Paprikafrüchte, welche aus der Umgegend von Szeged stammten, wurden bei einer Temperatur von beiläufig $110^{\circ} 6^{1 / 2}$ Stunden lang getrocknet und dann zusammen mit den Kelchen, Stengeln, Samen vermahlen und das Mahlgut in diesem Zustande untersucht.

B. Genau die gleichen wie unter A. beschriebenen Früchte wurden auseinander präpariert. Das abgetrennte Fruchtfleisch samt den inneren häutigen Teilen, jedoch ohne Samen, Kelche und Stengel, wurde 7 Stunden lang bei $105-108^{\circ} \mathrm{C}$ getrocknet und dann fein vermahlen und in diesem Zustande untersucht.

Die Bestimmung des Aschengehaltes in diesen beiden Proben lieferte folgende Ergebnisse :

$$
\begin{aligned}
& \text { A. }\left\{\begin{array}{c}
5,0733 \mathrm{~g} \text { gaben an Asche } 0,3581 \mathrm{~g}=7,05 \% \\
4,5148, \pi, \pi 0,3168,=7,01,
\end{array}\right\} \text { im Mittel } 7,03 \%
\end{aligned}
$$

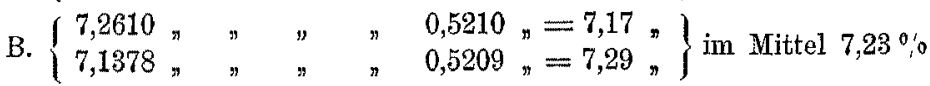

Bestimmt wurde somit der Aschengehalt von 20 Paprikaproben, deren Reinheit und Echtheit absolut keinen Zweifel aufkommen ließen, und aus den Untersuchungsergebnissen geht hervor, daß vollkommen reine, aus den Fruchtschalen, Samen und den Samenträgern gemahlene Paprikamuster 7-8\% Asche enthalten, welche Aschenmenge ganz und gar nicht auffallend ist. Wenn daher in einem Handelspaprikamuster ein Aschengehalt von 7-8\% gefunden wird und die nähere Untersuchung die Reinheit des Musters beweist, so ist dieser Umstand allein kein Beweis dafür, daß dieser Paprika gefälscht ist oder sonst irgendwie zu beanstanden wäre.

Vor dem Vermahlen wurden die Paprikaschoten mit Hilfe von Gänsefedern gut gereinigt, um von der Oberfläche der Schoten den Staub und die etwa anhängenden erdigen und sonstigen Teile zu entfernen. Um die Ausführung dieser Arbeit zu kontrollieren, wurde bei einigen der Muster der in 10\%-iger heißer Salzsäure unlösliche Rückstand (Sand) bestimmt, Hierbei wurden folgende Ergebnisse, auf $100 \mathrm{~g}$ Trockensubstanz berechnet, erhalten:

$\begin{array}{cccccc}\text { I } & \text { II } & \text { III } & \text { IV } & \text { V } & \text { VI } \\ 0,16 & 0,26 & 0,20 & 0,21 & 0,15 & 0,09 \%\end{array}$

Die Kelche und Stengel der Paprikafrüchte, welche vor dem Vermahlen von den Samen und dem Fruchtfleische getrennt wurden, sind ebenfalls zur Bestimmung des Aschengehaltes benutzt worden. Ihr Aschengehalt wurde in jenem Zustande (lufttrocken) bestimmt, wie sie in das Laboratorium kamen. Die darauf bezüglichen analytischen Daten sind ebenfalls in der Tabelle auf S. 392 u. 393 aufgeführt. Der niedrigste Aschengehalt war $10,71 \%$, der höchste 14,12\%, das Mittel aus sämtlichen Zahlen berechnet sich zu 12,09\%.

Die ersten 6 Muster enthielten an "Sand" in 100 Teilen:

$\begin{array}{cccccc}\text { I } & \text { II } & \text { III } & \text { IV } & \text { V } & \text { VI } \\ 0,33 & 0,29 & 0,35 & 0,21 & 0,33 & 0,48 \%\end{array}$


In den Jahrgängen 1904 und 1905 der „Zeitschrift für das landwirtschaftliche Versuchswesen in Öterreich" sind zwei Arbeiten ${ }^{1}$ ) von mir erschienen unter den Titeln „Studien über den Sandgehalt des Paprika“") und "Über den Sandgehalt des Paprika“" ${ }^{3}$ ). In diesen Arbeiten berichtete ich über die Ergebnisse meiner diesbezüglichen Arbeiten und machte gleichzeitig einen Vorschlag hinsichtlich der Beurteilung des Sandgehaltes des Paprikas ${ }^{4}$ ). In derselben Zeitschrift ist ferner unter dem Titel „Beiträge zur Beurteilung des Paprika" aus der Feder des Herrn O. v, Czadek eine Arbeit erschienen, in welcher er über meine oben erwähnten Arbeiten folgende Bemerkungen macht:

${ }_{n}$ Herr Dr.Windisch schlägt als oberste zulässige Grenze für den Sandgehalt des Paprikas $1,50 \%$ vor. Za diesem Werte ist er auf Grund einer größeren Reihe von Untersuchungen von gemahlenen im Handel befindlichen Paprikasorten gekommen. Von vorneherein will ich bemerken, dak ich es nicht für den richtigen Weg halte, auf Grund von Handelsprodukten, deren Herstellung man nicht überwachen kann, neue Grenzwerte für die Beurteilung der Ware aufzustellen. Herr Dr. Wind is ch hätte sich nach meiner Meinung zu diesem Zwecke ausschließlich der ganzen Früchte verschiedener Provenienz bedienen müssen, um ans den Ergebnissen dieser' Untersuchungen zr einem brauchbaren Resultate zu gelangen."

Es sei mir hier gestattet, auf die Bemerkungen des Herrn O. v. Czadek folgendes zu erwidern:

Wenn ich in dem gemahlenen Paprika des Handels einen Bestandteil bestimmen wollte, welcher einen normalen Bestandteil der Paprikafrüchte bildet z. B. den ätherischen oder alkoholischen Extrakt, Asche, Rohfaser etc. etc. und deren Menge zur Aufstellung von Grenzwerten benutzt worden wäre, in diesem Falle wäre unbedingt das durch Herrn $O$. $\vee$. Czadek empfohlene Vorgehen das einzig richtige und korrekte. Nämlich es müßten unbedingt selbstgemahlene oder aber unter strenger Kontrolle gemahlene Paprikapulver untersucht werden.

Bei den erwähnten Untersuchungen war jedoch der Zweck, einen zufälligen Bestandteil, die Menge des Sandes, zu bestimmen.

Wenn zu diesem Zweck selbstgemahlene Paprikapulver, etwa ganze Früchte untersucht und deren Sandgehalt bestimmt worden wäre, wäre es sehr leicht möglich gewesen, solche analytische Ergebnisse zu erhalten, welche man bei der Beurteilung der Handelspaprikasorten nicht hätte verwerten können.

Bei der Darstellung von gemahlenem Paprika, auch in geringer Quantität im Laboratorium, hätte man unbedingt die dazu dienenden Früchte reinigen müssen, teils um die darunter etwa vorhandenen fremden Bestandteile $\mathrm{zu}$ entfernen, teils um die den Früchten anhängenden erdigen und sandigen Teile zu beseitigen; um so mehr als bei der fabriksmäßigen Darstellung des Paprikas die zu vermahlenden Früchte wiederholt ausgewählt und gereinigt werden, sehr oft auch durch Waschen. Es unterliegt keinem Zweifel, daß, wenn man im Laboratorium nur einige Hunderte Gramm Paprikafrüchte sowohl vom Sande, als auch von sonstigen fremden Bestandteilen vollkommen reinigen will, dies ganz gewil viel vollkommener zu erreichen ist als bei der fabriksmäbigen Darstellung des Paprikas, wo die Früchte zentnerweise verarbeitet werden.

1) Ursprünglich in ungarischer Sprache ersehienen in den „Kisérletügyi Közlemények 1903.

$\left.{ }^{2}\right)$ Zeitschr. Landw. Versuchswesen in Österreich 1904, 7, 19.

3) Daselbst 1905, 8, 73.

4) Daselbst 1905, 8, 562 . 
Ich fand in durchaus eigenhändig gemahlenen Paprikapulvern folgende Sandmengen:
0,16
0,26
0,20
$0,21 \quad 0,15$
$\left.0,09^{1}\right)$
$\left.0,12^{2}\right)$
$0,04 \%$

Bei meinen oberwähnten Untersuchungen wollte ich bestimmen, inwiefern es möglich ist und auch gelingt, bei der fabriksmäBigen Aufarbeitung der Paprikaschoten dieselben vom Sande zu reinigen; dies war aber auf andere Weise nicht zu erreichen, als wie ich seinerzeit vorgegangen bin. Die zur Beurteilung des Sandgehaltes aufgestellten Grenzwerte waren nicht nur vom einseitigen Standpunkte des Konsumenten und kaufenden Publikums zu beurteilen, sondern man mußte auch auf die Fabrikanten Rücksicht nehmen. Der ungarische Gesetzartikel No. 46 rom Jahre 1895 ist berufen, die Interessen sowohl des Konsumenten als auch gleichzeitig des Fabrikanten zu wahren.

In acht selbstgemahlenen Paprikapulvern, welche aus acht verschiedenen Sorten Paprika dargestellt wurden, fand ich im Mittel 0,15\% Sand. Wenn diese Analysenergebnisse dazu benutzt worden wären, um Grenzwerte betreffs des erlaubten oder normalen Sandgehaltes aufzustellen, würde dies sehr leicht zu einem Irrtum oder Fehler geführt haben, welcher ohne einen gewichtigen Grund den Fabrikanten Unannehmlichkeiten verursacht hätte, oder aber es wäre auf Grund nur dieser Ergebnisse der normierte Sandgehalt durch die Fabrikanten bezw. die Fabrikationsweise überhaupt nicht zu erfüllen gewesen.

Als B. Schulze ${ }^{3}$ ) einen Vorschlag behufs Beurteilung des normalen Sandgehaltes der verschiedenen Hundelsfuttermittel machen wollte, war sein Vorgehen folgendes: Er bestimmte den Sandgehalt von $1100 \mathrm{im}$ Handel vorkommenden verschiedenen Futtermitteln. Bei Besprechung seiner Untersuchungsergebnisse kommt er zu dem Resultate, daß bei den verschiedenen Futtermitteln nicht die gleiche Menge Sand als normal anzunehmen ist, und er führt gruppenweise diejenigen Futtermittel. an, in welchen eine Sandmenge von $0,5,0,8,1,0,1,50 \%$ als normales Maximum anzunehmen ist. Das Vorgehen Schulze's war, da es sich um die Bestimmung eines zufälligen Bestandteiles handelte, richtig; es wäre dagegen unrichtig gewesen, wenn er die Untersuchungsergebnisse selbstverfertigter Futtermittel zur Aufstellung von Grenzwerten benutzt hätte.

Herr O. v. Czadek wendet sich ferner gegen die von mir verlangte Heraufsetzung der Sandgrenze auf $1,50 \%$, da in seiner Ware $1 \%$ fast niemals erreicht wurde.

Mein Vorschlag ${ }^{4}$ ) zur Beurteilung des Sandgehaltes lautete folgendermaBen:

„Ein Gehalt von 0,5\% Sand in dem Mahlprodukte der Paprikasehoten kann als normaler Sandgehalt angenommen werden. Ist der Sandgehalt höher wie $0,50 \%$ jedoch geringer als $1,50 \%$, würde die Ware als unrein (sandig) anzusprechen sein. Bei einem höheren Sandgehalte wie $1,50 \%$ wäre die Ware zu beanstanden."

In Österreich haben die Versuchsstationen als Grenze für den Sandgehalt $1 \%$ angenommen und jede Ware, welche mehr als $1 \%$ Sand enthält, wird beanstandet.

1) Fruchte, welche mitsammt den Kelchen and Stengeln vermahlen wurden.

2) Nar durch Vermahlen des roten Fruchtfleisches und der Samenträger dargestellt.

3) B. Schulze: Studien über den Sandgehalt der Handelsfuttermittel. (Laudw. Versucbsstationen 1896, 47, 361.)

4) Zeitschrift für das landwirtschaftliche Versuchswesen in Österreich 1904, 8, 20. 
Nach meinem Vorschlage kann ein Sandgehalt bis $0,5 \%$ als normal bezeichnet werden; Sand bis zu dieser Grenze enthaltende Ware kann daher als normale Ware angenommen werden und kann allein auf Grund dieses Sandgehaltes nicht beanstandet werden. Eine Ware, welche mehr als $0,5 \%$ jedoch weniger wie $1,50 \%$ Sand enthält, wäre als unrein (sandig) zu bezeichnen. Ist jedoch der Sandgehalt höher wie $1,50 \%$, so wäre die Ware zu beanstanden. In Österreich dagegen wird bis zu einem Sandgehalt von 1\% gar kein Einwand erhoben, während nach meinem Vorschlag eine mehr als $0,5 \%$ Sand enthaltende Ware schon als un rein bezeichnet wird. SchlieBlich aber hielt ich mich für die Beantwortung der Frage, wie überhaupt der Sandgehalt beurteilt werden soll, allein nicht für berufen und darum waren meine diesbezüglichen Bemerkungen nur in der Form eines Vorschlages veröffentlicht.

\section{Polarimetrische Bestimmung der Znckerarten im Honig.}

\section{Von}

\section{Dr. Paul Lehmann und Dr. Hermann Stadlinger in Erlangen.}

Ein interessanter Prozeß, gleich bedeutsam für den Nahrungsmittelchemiker wie für den praktischen Imker, spielte sich unlängst vor dem Gerichtshofe einer süddeutschen Stadt ab. Zwei Inhaber weit bekannter Honigversandgeschäfte hatten sich wegen unlauteren Wettbewerbes, begangen dadurch, daß sie große Mengen geläuterten Havannahonigs mit Hilfe entsprechender Zeitungsinserate als "Schleuderhonig, garantiert reines und unverfälschtes Naturprodukt vorzüglichster Qualität", in den Handel gebracht hatten, zu verantworten.

Gegen einen der beiden Honighändler war außerdem wegen $\mathrm{Nahrungsmittel.}$ fälschung Anklage erhoben, wonach er ,seit 1902 verschiedenfach echten Honig mit Kunsthonig vermischt und dieses so verfälschte Produkt unter Verschweigung der Verfälschung als Schleuderhonig, garantiert reines und unverfälschtes Naturprodukt, verkauft und feilgehalten" hatte.

Der ProzeB, der mit einem Freispruch der Angeklagten endete, hat bereits von mehreren Seiten eine kritische Beleuchtung gefunden, so daß wir davon absehen können, den ersten Punkt der Anklage in den Bereich unserer Erörterungen zu ziehen, umsomehr, als es sich hierbei um Fragen handelt, die weit mehr die Interessen der Bienenzüchter als diejenigen des Chemikers berühren. Weit wichtiger erscheint uns der zweite Anklagepunkt, die Verfälschung von Bienenhonig mit Saccharosesirup, zu deren Klarlegung sich $\operatorname{der}$ Eine $^{1}$ ) von uns neben einer Reihe anderer Sachverständigen vor Gericht gutachtlich zu äußern hatte.

Als Grundlage für die Anklage dienten die nachfolgend verzeichneten 8 Analysen, die dem Laboratorium eines in Imkerkreisen hochangesebenen Chemikers entstammten. Genannte Proben wurden teils von der Gerichtskommission unter Zuziehung eines Sachverständigen aus der Imkerpraxis in Geschäftslokale des Angeklagten entnommen, teils entstammten sie einer Postsendung, deren Inbalt ein Kunde des Angeklagten unter Garantie der Reinheit empfangen hatte.

1) Stadlinger: 